\title{
Correction to: Biosynthesis of pneumocandin lipopeptides and perspectives for its production and related echinocandins
}

\author{
Yan $\mathrm{Li}^{1} \cdot \mathrm{Nan}_{\mathrm{Lan}}{ }^{2} \cdot \mathrm{Lijian}_{\mathrm{Xu}}{ }^{3} \cdot \mathrm{Qun} \mathrm{Yue}^{4}$ \\ Published online: 16 September 2021 \\ (c) Springer-Verlag GmbH Germany, part of Springer Nature 2021
}

\section{Correction to: Applied Microbiology and Biotechnology (2018) 102:9881-9891 https://doi.org/10.1007/s00253-018-9382-x}

The original version of this article contains errors.

In Fig. 1a, L-leucine should be cyclized by GLOXY4 to form 4-methyl-L-proline, which should be then catalyzed by GLOXY2 to form 3-hydroxy-4-methyl-L-proline. L-proline should be also catalyzed by GLOXY2 to form 3-hydroxyL-proline. Fig. $1 \mathrm{~b}$, in rows " $\mathrm{B}_{1}$ " and " $\mathrm{A}_{1}$ " column 3 , the $\mathrm{R}_{1}$ positions of pneumocandins $\mathrm{B}_{1}$ and $\mathrm{A}_{1}$ should be corrected from " $\mathrm{OH}$ " to "H".

The corrected version of this figure is shown below.

Publisher's note Springer Nature remains neutral with regard to jurisdictional claims in published maps and institutional affiliations.

The original article can be found online at https://doi.org/10.1007/ s00253-018-9382-x.

\section{Lijian Xu}

xulijian@hlju.edu.cn

$\triangle$ Qun Yue

yuequn@caas.cn

1 Institute of Vegetables and Flowers, Chinese Academy of Agricultural Sciences, Beijing 100081, China

2 Texas Therapeutics Institute, The Brown Foundation Institute of Molecular Medicine, University of Texas Health Science Center at Houston, Houston, TX 77054, USA

3 College Agricultural Resource and Evironment, Heilongjiang University, 74 Xuefu Road, Harbin 150080, China

4 Biotechnology Research Institute, Chinese Academy of Agricultural Sciences, Beijing 100081, China 
a

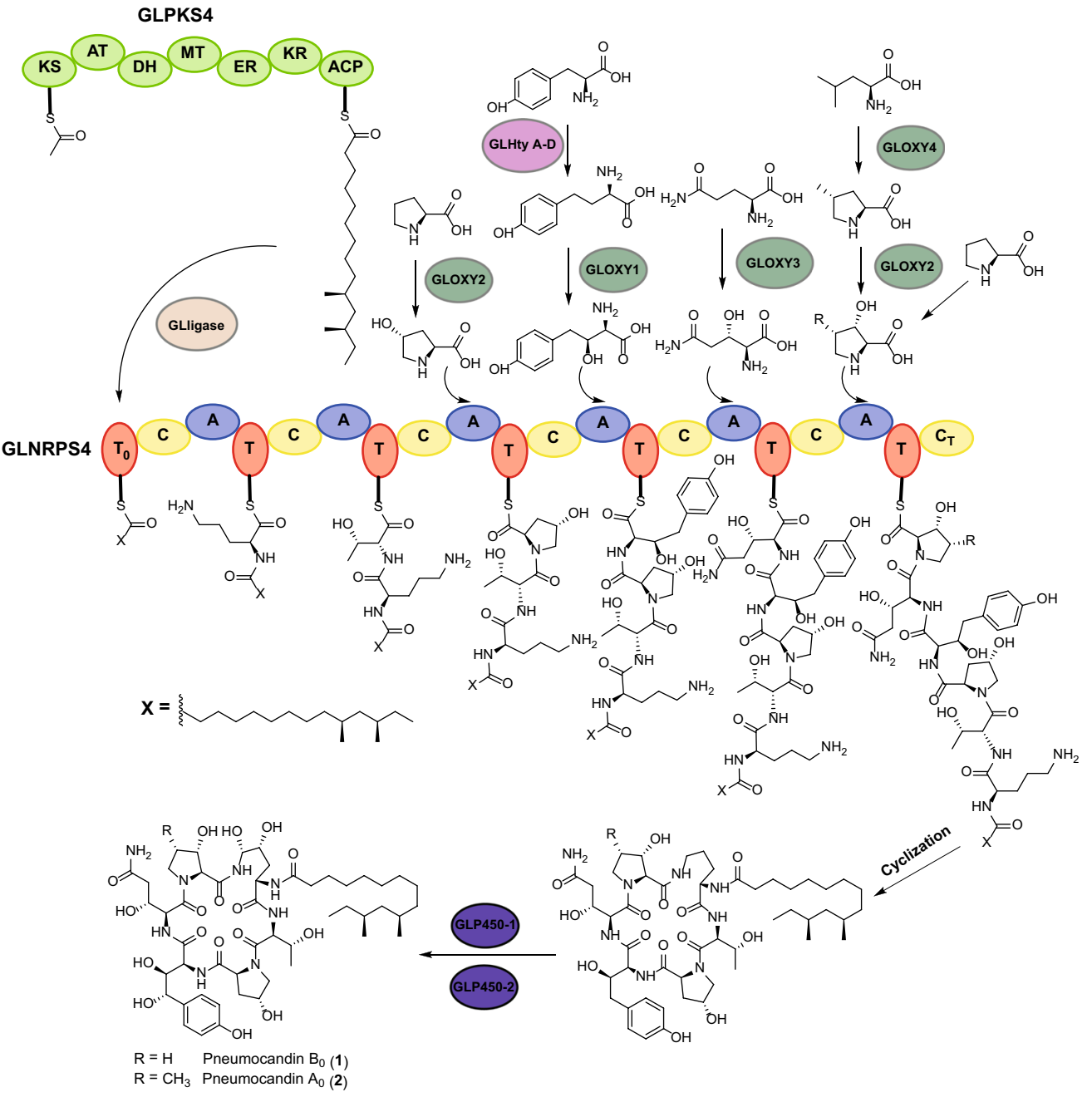

b

\begin{tabular}{|c|c|c|c|c|c|c|}
\hline & Pneumocandins & $x$ & $R_{1}$ & $\mathrm{R}_{2}$ & $\mathrm{R}_{3}$ & $R_{4}$ \\
\hline & $\mathrm{A}_{0}$ & 10,12-dimethy Imy ristic acid & $\mathrm{OH}$ & $\mathrm{OH}$ & $\mathrm{OH}$ & $\mathrm{CH}_{3}$ \\
\hline & $\mathrm{B}_{0}$ & 10,12-dimethy Imyristic acid & $\mathrm{OH}$ & $\mathrm{OH}$ & $\mathrm{OH}$ & $\mathrm{H}$ \\
\hline & $\mathrm{A}_{2}$ & 10,12 -dimethy Imy ristic acid & $\mathrm{OH}$ & $\mathrm{H}$ & $\mathrm{H}$ & $\mathrm{CH}_{3}$ \\
\hline & $\mathrm{B}_{2}$ & 10,12 -dimethy Imy ristic acid & $\mathrm{OH}$ & $\mathrm{H}$ & $\mathrm{H}$ & $\mathrm{H}$ \\
\hline & $\mathrm{F}$ & 10,12-dimethy Imy ristic acid & $\mathrm{H}$ & $\mathrm{OH}$ & $\mathrm{OH}$ & $\mathrm{H}$ \\
\hline & G & 10,12-dimethy Imyristic acid & $\mathrm{H}$ & $\mathrm{OH}$ & $\mathrm{OH}$ & $\mathrm{CH}_{3}$ \\
\hline & $\mathrm{B}_{1}$ & 10,12-dimethy Imy ristic acid & $\mathrm{H}$ & $\mathrm{OH}$ & $\mathrm{OH}$ & $\mathrm{H}$ \\
\hline \multirow[t]{13}{*}{ Pneumocandins } & $A_{1}$ & 10,12-dimethy Imy ristic acid & $\mathrm{H}$ & $\mathrm{OH}$ & $\mathrm{OH}$ & $\mathrm{CH}_{3}$ \\
\hline & $\mathrm{A}_{3}$ & 10,12-dimethy Imy ris tic acid & $\mathrm{H}$ & $\mathrm{H}$ & $\mathrm{OH}$ & $\mathrm{CH}_{3}$ \\
\hline & $\mathrm{A}_{4}$ & 10,12-dimethy Imy ristic acid & $\mathrm{H}$ & $\mathrm{H}$ & $\mathrm{H}$ & $\mathrm{CH}_{3}$ \\
\hline & 9 & 10,12-dimethy Imy ristic acid & $\mathrm{OH}$ & $\mathrm{H}$ & $\mathrm{OH}$ & $\mathrm{CH}_{3}$ \\
\hline & 10 & 10,12 -dimethy Imy ristic acid & $\mathrm{H}$ & $\mathrm{H}$ & $\mathrm{OH}$ & $\mathrm{H}$ \\
\hline & 11 & 10,12-dimethy Imyristic acid & $\mathrm{H}$ & $\mathrm{OH}$ & $\mathrm{H}$ & $\mathrm{H}$ \\
\hline & 13 & 10,12-dimethy Imyristic acid & $\mathrm{H}$ & $\mathrm{OH}$ & $\mathrm{H}$ & $\mathrm{CH}_{3}$ \\
\hline & 14 & 10,12-dimethy Imy ristic acid & $\mathrm{H}$ & $\mathrm{H}$ & $\mathrm{H}$ & $\mathrm{H}$ \\
\hline & $\mathrm{H}$ & myristic acid & $\mathrm{OH}$ & $\mathrm{OH}$ & $\mathrm{OH}$ & $\mathrm{H}$ \\
\hline & 1 & pentadecanoic acid & $\mathrm{OH}$ & $\mathrm{OH}$ & $\mathrm{OH}$ & $\mathrm{CH}_{3}$ \\
\hline & $\mathrm{J}$ & palmitic acid & $\mathrm{OH}$ & $\mathrm{OH}$ & $\mathrm{OH}$ & $\mathrm{H}$ \\
\hline & $\mathrm{K}$ & palmitic acid & $\mathrm{OH}$ & $\mathrm{OH}$ & $\mathrm{OH}$ & $\mathrm{CH}_{3}$ \\
\hline & Acrophiarin & myristic acid & $\mathrm{OH}$ & $\mathrm{OH}$ & $\mathrm{OH}$ & $\mathrm{CH}_{3}$ \\
\hline
\end{tabular}

